\title{
Adaptive Sliding Mode Contouring Control Design Based on Reference Adjustment and Uncertainty Compensation for Feed Drive Systems
}

\author{
Mathew Renny Msukwa* and Santos Kihwele \\ Department of Electrical Engineering, University of Dar es Salaam, P. O. Box 35131, Dar es \\ Salaam, Tanzania \\ E-mail addresses: rennymatt@gmail.com; kihwele2002@yahoo.com \\ *Corresponding author
}

Received 13 Sep 2021, Revised 2 Dec 2021, Accepted 6 Dec 2021, Published Dec 2021

DOI: https://dx.doi.org/10.4314/tjs.v47i5.23

\begin{abstract}
Industrial feed drive systems, particularly, ball-screw and lead-screw feed drives are among the dominating motion components in production and manufacturing industries. They operate around the clock at high speeds for coping with the rising production demands. Adversely, high-speed motions cause mechanical vibrations, high-energy consumption, and insufficient accuracy. Although there are many control strategies in the literature, such as sliding mode and model predictive controls, further research is necessary for precision enhancement and energy saving. This study focused on design of an adaptive sliding mode contouring control based on reference adjustment and uncertainty compensation for feed drive systems. A combined reference adjustment and uncertainty compensator for precision motion of industrial feed drive systems were designed. For feasibility of the approach, simulation using matlab was conducted, and results are compared with those of an adaptive nonlinear sliding model contouring controller. The addition of uncertainty compensator showed a substantial improvement in performance by reducing the average contour error by $85.71 \%$ and the maximum contouring error by $78.64 \%$ under low speed compared to the adaptive sliding mode contouring controller with reference adjustment. Under high speed, the addition of uncertainty compensator reduced the average and absolute maximum contour errors by $4.48 \%$ and $10.13 \%$, respectively. The experimental verification will be done in future.
\end{abstract}

Keywords: Machine tools, Feed drive systems, contouring control, Uncertainty dynamics, Sliding mode control.

\section{Introduction}

Feed drive systems are among the most dominating motion components in the production and manufacturing industries because of their wide range of use (e.g., in multi-axis motions) (Xu et al. 2017, Simba et al. 2018, Zhu and Farouki 2019, Nshama et al. 2021). The growing demands for precise products pose the need for high-speed production systems with high accuracy. In addition, feed drive systems operate around the clock; therefore, they are among the major consumers of the industrial energy supply, particularly the manufacturing sector is estimated to deplete about one-third of the world's energy consumption (Yoon 2015). The energy consumption of machine tool is attracting more attention because also of the increase of energy prices (Ji et al. 2020).

While high-speed motion is preferred, it causes vibrations in light systems, highenergy consumption, and a poor tracking performance. As explained in Simba et al. (2018) and Dong and Tang (2014), the control performance greatly depends on the system vibration, unmodeled uncertainties, and 
external disturbances in practical applications.

In machining, two main control approaches are used to enhance precision: tracking control approach and contouring control approach. Although many approaches for reducing the tracking errors in feed drive systems have been developed to date (Msukwa et al. 2017, Msukwa et al. 2019, Farrage and Uchiyama 2020), the most significant factor is the overall system accuracy or the system contour error (Ramesh et al. 2005, Hu et al. 2010). Contouring control is a controller design that considers the error components orthogonal to the desired contour curves, called "contour errors," as feedback signals.

A variety of alternative approaches have been developed to reduce the contour errors. By calculating the contour errors from the tracking errors in biaxial contour-following tasks, the authors in Koren and Yoram (1980) proposed the Cross-coupled biaxial Computer Control (CCC) for manufacturing systems, while those in Ho et al. (1999) decomposed the contour errors into the normal tracking and advancing tangential errors. Dynamic decoupling was then applied to the system dynamics and contour accuracy was improved. In Yeh and Hsu (2002), the contour error was estimated as the vector from the actual position to the tangential line at the reference position. The authors in Chen et al. (2002) proposed a contour-tracking controller based on polar coordinates. When using CCC methods, one of the advantages is that both the contour and tracking errors along the feed drive axes are used to calculate the control input, which causes degradation in the contour-tracking performance.

Robust controllers, such as the sliding mode controller (SMC), provide a reasonable performance under the effect of external disturbance and system uncertainties (Raman et al. 2013, Dong and Tang 2014, Mohammad et al. 2015, Zheng et al. 2015, Xi et al. 2015, Yang and Altintas 2015, Li et al. 2016). Apart from its simplicity in design, SMC is robust against perturbations and invariant to matched uncertainties. Other SMC variants include adaptive sliding mode control (ASMC) and nonlinear sliding mode control (Sencer and
Shamoto 2013, Chen et al. 2016, Farrage and Uchiyama 2019).

Meanwhile, model-based approaches, such as feedforward friction compensation, are applied to cancel out the effect of the estimated friction force. However, friction sources generally consist of complex nonlinear properties such as Coulomb friction which is zero at zero speed and has a fixed value at all non-zero speeds; hence, finding an exact model is difficult, and the performance exclusively depends on the veracity of the estimated model (Elfizy et al. 2004, Kamalzadeh et al. 2010).

This study focused on developing an adaptive sliding mode contouring control (ASMCC) for feed drive systems, whose main objective was to enhance the contouring performance by explicitly considering reference adjustments with addition of an uncertainty dynamics compensator. Nonlinear SMC and an additional control input were designed to compensate for the uncertainty dynamics. Accordingly, this was done by modeling the assumed uncertainty dynamics (see the uncertainty compensation section). The uncertainties controller was designed by taking the difference between a reference model and the real system based on the idea presented in Kayacan and Peschel (2016). The proposed method was found to enhance both the tracking and contouring performances of feed drive systems while maintaining the required energy. The system stability was analyzed and confirmed through the Lyapunov theory. In addition, its convergence to the sliding surface was assured.

\section{Methods}

\section{Contour error estimation}

The proposed control structure comprises of a contouring controller, actual system, reference model, and an uncertainty dynamics compensator as shown in Figure 1. This subsection explains the contouring control with a typical biaxial setup (i.e., X-Y table system), assuming the following dynamics:

$$
u=M \ddot{q}+C \dot{q}+L \operatorname{sign}(\dot{q})+d
$$

where $q=[x, y]^{T}$, and $M=\operatorname{diag}\left(m_{x}, m_{y}\right)$, $C=\operatorname{diag}\left(c_{x}, c_{y}\right), \quad L=\operatorname{diag}\left(l_{x}, l_{y}\right), \quad d=$ 
$\left[d_{x}, d_{y}\right]^{T}$, and $u=\left[u_{x}, u_{y}\right]^{T}$ are the position of the feed drive system, mass matrix, viscous coefficient matrix, Coulomb friction matrix, disturbance vector, and control input vector consisting of each axial element, respectively. The following linear equation is considered as the reference model:

$$
\bar{u}=M \ddot{\ddot{q}}+C \dot{\bar{q}}
$$

where $\bar{q}=\left[\bar{q}_{x}, \bar{q}_{y}\right]^{T}$ and $\bar{u}=\left[\bar{u}_{x}, \bar{u}_{y}\right]^{T}$ are the position of the reference plant model and the input vector, respectively. The control input to the real plant is defined as $u=\bar{u}+v$, where $v=\left[v_{x}, v_{y}\right]^{T}$ is the compensator for the uncertainty dynamics. In machining, the tracking error reduction for each feed drive axis is not as necessary as reducing the contour error (Uchiyama et al. 2009). The contour error is more important because it is directly related to the shape of the machined part in the machining application. Therefore, the proposed contouring control has a degree of freedom (DOF) tangent to the contour curve, and this DOF can be exploited for energy saving.

Figure 2 schematically explains the relationship between the tracking error on each axis and the contour error. The coordinate frame $\sum_{\omega}$ is a fixed frame with $x$ and $y$ axes corresponding to the feed drive axes. Curve $C$ represents the desired contour curve of the point of a feed drive. The reference trajectory, $r=\left[r_{x}, r_{y}\right]^{T}$ is the desired position vector of the point of the feed drive at time $t$ defined in the coordinate frame $\sum_{\omega}$. The second derivative is required in the controller design; thus, the reference trajectory $r$ is assumed to have $C^{2}$ continuity. Like the reference trajectory $r$, the real position of the feed drive $q$ is also defined in $\sum_{\omega}$. The tracking error vector $e_{\omega}$ of the system is given as

$$
e_{\omega}=\left[e_{\omega x}, e_{\omega y}\right]^{T}=q-r .
$$

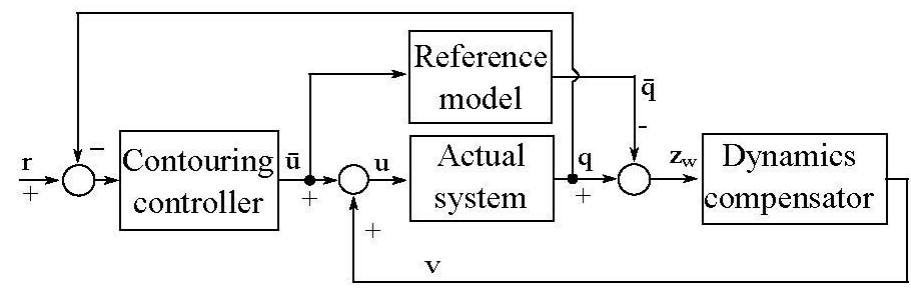

Figure 1: Block diagram of the proposed ASMCC control system.

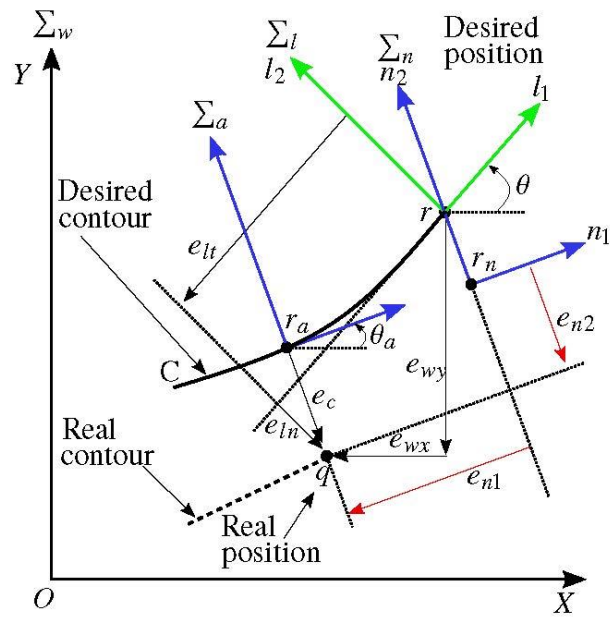

Figure 2: Definitions of tracking errors.
Feed drives are generally controlled to minimize the magnitude of the tracking errors by independently controlling the drive axes. The contour error $e_{c}$ is defined as the shortest distance between $q$ and $r_{a}$. This distance is the shortest distance from $q$ to the desired curve $C$. In machining, the contour error $e_{c}$, rather than the tracking error $e_{\omega}$, should be minimized because $e_{c}$ is directly related to the shape of the machined part. In other words, if $e_{c}$ is minimized to zero, the desired shape of the machined part can be realized, even though the tracking error $e_{\omega}$ remains. The following error coordinate transformation is typical in contouring control (Uchiyama et al. 2015(a)): 


$$
\begin{aligned}
e_{l}=\left[e_{l t}, e_{l n}\right]^{T}= & R^{T} e_{\omega}, R \\
& =\left[\begin{array}{cc}
\cos \theta & -\sin \theta \\
\sin \theta & \cos \theta
\end{array}\right],
\end{aligned}
$$

where $\theta$ is the inclination of $\sum_{l}$ to $\sum_{\omega}$ (Figure 2 ), and $e_{l t}$ and $e_{l n}$ are the tangential and normal error components to the desired contour curve at position $r$, respectively.

The time $t_{d}$ for the trajectory to pass from $r$ to $r_{a}$ is approximated as:

$$
t_{d}=-\frac{e_{l t}}{\sqrt{\dot{r}_{x}^{2}+\dot{r}_{y}^{2}}}
$$

The estimation of $r_{a}$ and the inclination $\theta_{a}$ of frame $\sum_{a}$ in Figure 2 are presented as follows (Uchiyama et al. 2015a):

$$
\hat{r}_{a}=r\left(t-t_{d}\right), \hat{\theta}_{a}=\theta\left(t-t_{d}\right),
$$

where $\hat{r}_{a}$ and $\hat{\theta}_{a}$ are the estimates of $r_{a}$ and $\theta_{a}$, respectively. $r()$ and $\theta()$ denote the functions of time. The modified desired position $r_{n}$ is represented from Figure 2 as

$$
\begin{gathered}
r_{n}=r+Q\left(\hat{r}_{a}-r\right), Q=\hat{R}_{a} S \hat{R}_{a}^{T}, S \\
=\left[\begin{array}{ll}
0 & 0 \\
0 & 1
\end{array}\right],
\end{gathered}
$$

where $\hat{R}_{a}$ is an approximation of a rotation matrix at $\sum_{a}$. Matrix $\hat{R}_{a}$ was obtained by replacing angle $\theta$ in $R$ in (4) with $\hat{\theta}_{a}$. The first and second derivatives of $r_{n}$ are presented below.

$$
\begin{gathered}
\dot{r}_{n}=\dot{r}+\dot{Q}\left(\hat{r}_{a}-r\right)+Q\left(\dot{\hat{r}}_{a}-\dot{r}\right) \\
\ddot{r}_{n}=\ddot{r}_{n}+\ddot{Q}\left(\hat{r}_{a}-r\right)+2 \dot{Q}\left(\dot{\hat{r}}_{a}-\dot{r}\right) \\
+Q\left(\ddot{\hat{r}}_{a}-\ddot{r}\right) .
\end{gathered}
$$

By taking the time derivative of the relation $e_{n}=\hat{R}_{a}^{T} e_{\omega n}$, where $e_{n}=\left[e_{n 1}, e_{n 2}\right]^{T}$, we obtain the following Equation (9):

$$
\begin{gathered}
\dot{e}_{n}=\hat{R}_{a}^{T} \dot{e}_{w n}+\dot{\hat{R}}_{a}^{T} e_{w n} \\
\ddot{e}_{n}=\hat{R}_{a}^{T} \ddot{e}_{w n}+2 \dot{\hat{R}}_{a}^{T} \dot{e}_{w n}+\ddot{\hat{R}}_{a}^{T} e_{w n}=\hat{R}_{a}^{T}\left(\ddot{e}_{w n}+\dot{\hat{\theta}}_{a} I_{e} e_{w n}-\dot{\hat{\theta}}_{a}^{2} I e_{w n}+2 \dot{\hat{\theta}}_{a} I_{e} \dot{e}_{w n}\right), \\
I_{e}=\left[\begin{array}{cc}
0 & 1 \\
-1 & 0
\end{array}\right],
\end{gathered}
$$

where $I$ is a $2 \times 2$ identity matrix, and the properties of rotation matrix $\hat{R}_{a} \ddot{\hat{R}}_{a}^{T}=\ddot{\hat{\theta}}_{a} I_{e}-\dot{\hat{\theta}}_{a}^{2} I$ and $\hat{R}_{a} \dot{\hat{R}}_{a}^{T}=\dot{\hat{\theta}}_{a} I_{e}$ are applied.

\section{Adaptive sliding mode contouring controller design}

Two steps are normally involved when designing a sliding mode controller. We first considered the selection of a nonlinear sliding surface, in which the system tracks a reference trajectory. The sliding surface should ensure that the control system dynamics is exponentially stable, such that a control law that drives the system to track the desired trajectory in finite time can be designed. The dynamic system response depends on its damping ratio (Bandyopadhyay et al. 2009). Preferably, one must have a system that responds as fast as possible without overshoots. At the initial stage, a low damping ratio is applied to achieve a fast response. This ratio is then gradually increased to minimize overshoots. The advantage of using the variable damping ratio is that it provides a better tracking performance while reducing the energy consumption. The following nonlinear sliding surface is considered herein Mohammad et al. (2014):

$$
\begin{aligned}
\bar{s}=\left[\begin{array}{ll}
A & I
\end{array}\right]\left[\begin{array}{l}
e_{n} \\
\dot{e}_{n}
\end{array}\right], A & \\
& =\operatorname{diag}\left(\lambda_{j}+\psi_{j} \gamma_{j}\right), j \\
& =\{1,2\},
\end{aligned}
$$

where $\bar{s}=\left[\bar{s}_{1}, \bar{s}_{2}\right]^{T}$ is the nonlinear sliding variable vector, and $\lambda_{j}$ is the linear term of the sliding surface. The linear term is selected by ensuring that predominant poles have a small damping ratio. The $\gamma_{j}$ is a positive linear term used to adjust the damping ratio, while $\psi_{j}$ is a non-negative differentiable nonlinear function of the contour error, which is upper bounded, such that $\psi_{j} \leq \psi_{\max _{j}}$. Its role is to change the damping ratio of the system. Accordingly, $\psi_{j}$ gradually increased the damping ratio as the contour error decreased. The following 
function was considered herein based on the nonlinear function presented in $\mathrm{Su}$ et al. (2005) for a step-type reference trajectory:

$$
\begin{aligned}
& \psi_{j}=\beta_{j} \frac{\exp \left(-\bar{k}_{j} \tilde{e}_{n j}\right)+\exp \left(\bar{k}_{j} \tilde{e}_{n j}\right)}{2}, \\
& \tilde{e}_{n j} \\
& =\left\{\begin{array}{cl}
e_{n j} & \left|e_{n j}\right| \leq e_{n_{\max j}} \\
e_{n_{\max }} \operatorname{sign}\left(e_{n j}\right), & \left|e_{n j}\right|>e_{n_{\max j}}
\end{array}\right.
\end{aligned}
$$

where $\beta_{j}, \bar{k}_{j}$, and $e_{n_{\max }}$ are the positive tuning parameters defined by the controller designer, and $\beta_{j}$ and $\bar{k}_{j}$ determine the final damping ratio and the variation rate of the nonlinear function $\psi_{j}$, respectively. The magnitude of $\psi_{j}$ becomes small if the system output is far from the desired value. This provides a low damping ratio and speeds up the system response. On the sliding surface, $\bar{s}=0$,

$$
\dot{e}_{n}=-A e_{n},
$$

where $A$ was not a constant matrix. The following Lyapunov function was considered to verify the stability of the sliding surface, $\dot{\bar{S}}=0$ :

$$
V=\frac{1}{2} e_{n}^{T} e_{n}
$$

Substituting (12) into the time derivative of $V$ leads to:

$$
\dot{V}=-e_{n}^{T} A e_{n}
$$

The $A$ is a positive definite matrix; thus, the asymptotic stability is guaranteed.

The control law is designed such that from any initial condition, the reference model trajectory is attracted toward the sliding surface. The following control law is designed based on the proposed sliding surface and the feed drive dynamics:

$$
\begin{aligned}
& \bar{u}=M\left\{\ddot{r}_{n}-\hat{R}_{a}\left(A \dot{e}_{n}+B e_{n}+\widehat{K} \bar{s}\right)-\right. \\
& \left.\ddot{\hat{\theta}}_{a} I_{e} e_{w n}+\dot{\hat{\theta}}_{a}^{2} I e_{w n}-2 \dot{\hat{\theta}}_{a} I_{e} \dot{e}_{w n}\right\}+C \dot{q}+ \\
& L \operatorname{sign}(\dot{q}), B=\operatorname{diag}\left(\dot{\psi}_{j} \gamma_{j}\right), \widehat{K}= \\
& \operatorname{diag}\left(\hat{k}_{j}\right),
\end{aligned}
$$

where $L$ is a nominal value of Coulomb friction and $\hat{k}_{j}$ is the adaptive gain. Only the typical friction compensation was considered because of the difficulty in identification and the adaption and uncertainty dynamics compensation of other small disturbances. The adaption law was chosen based on the idea presented in Plestan et al. (2010):

$\dot{\hat{k}}_{j}=\left\{\begin{array}{cc}\xi_{j}\left|\bar{s}_{j}\right| \operatorname{sign}\left(\left|\bar{s}_{j}\right|-\varepsilon_{j}\right), & \text { if } \hat{k}_{j}>\zeta_{j} \\ \zeta_{j} & \text { otherwise }\end{array}\right.$,

where $\varepsilon_{j}, \zeta_{j}$, and $\xi_{j}$ are positive constants. Parameter $\zeta_{j}$ was introduced to obtain positive values for $\hat{k}_{j}$. After the sliding mode with respect to $\bar{s}_{j}$ was established, the gain adaption law (16) allowed the gain $\hat{k}_{j}$ to decrease while $\left|\bar{s}_{j}\right|<\varepsilon_{j}$. In other words, gain $\hat{k}_{j}$ will remain at the smallest level while satisfying the required accuracy of $\overline{s_{j}}$.

\section{Uncertainty compensation}

The real plant was different from the reference plant model; therefore, a controller was designed to compensate for the resulting uncertainty, which was eventually determined as the difference between the real plant and the reference plant model. Similar to the actual position of the real plant, the reference plant model was also defined in $\sum_{\omega}$. The uncertainty states are defined as follows:

$$
z_{w}=q-\bar{q},
$$

where $z_{w}=\left[z_{x}, z_{y}\right]^{T}$ is the measurable vector in Figure 1. With respect to $\sum_{n}$ in Figure 2, the uncertainty states are expressed as $z_{n}=\hat{R}_{a}^{T} z_{w n}, \quad$ where $z_{n}=\left[z_{n 1}, z_{n 2}\right]^{T}$ and $z_{w n}=z_{w}$ when $r_{n}$ is used as the desired position. The uncertainty dynamics was assumed as a second-order nonlinear dynamics as follows:

$$
\ddot{z}_{n}=\sigma\left(z_{n}\right)+v \text {, }
$$

where $v=\left[v_{x}, v_{y}\right]^{T}$ is the control input signal of the uncertainty dynamics, and $\sigma\left(z_{n}\right)=$ $\left[\sigma_{1}, \sigma_{2}\right]^{T}$ is the unknown time-varying dynamics of the system. 
The $\sigma$ was assumed to be upper-bounded by $\sigma_{\max }$ as:

$$
\left|\sigma_{j}\right| \leq \sigma_{\text {jmax }}
$$

The tracking error for the uncertainty dynamics is defined as follows:

$$
\tilde{z}_{w n}=z_{w n}-z_{r w n}, \quad \tilde{z}_{n}=\hat{R}_{a}^{T} \tilde{z}_{w n},
$$

where $\tilde{z}_{w n}=\left[\tilde{z}_{w n 1}, \tilde{z}_{w n 2}\right]^{T}$ and $\tilde{z}_{n}=$ $\left[\tilde{z}_{n 1}, \tilde{z}_{n 2}\right]^{T}$ are the tracking error vectors in the $\sum_{\omega}$ and $\sum_{n}$ coordinate frames, respectively, and $\tilde{z}_{r w n}=\left[\tilde{z}_{r w n 1}, \tilde{z}_{r w n 2}\right]^{T}$ is the desired position vector of the uncertainty dynamics. The control objective was to converge the output of the uncertainty dynamics to zero; thus, the desired value for the uncertainty position, velocity, and acceleration were set to 0 .

A linear SMC was used to cancel out the uncertainties. The following sliding surface $s=\left[s_{1}, s_{2}\right]^{T}$ consisting of the uncertainty error and the uncertainty error rate was used as:

$$
s=\alpha \tilde{z}_{n}+\dot{\tilde{z}}_{n}
$$

where $\alpha=\operatorname{diag}\left(\alpha_{1}, \alpha_{2}\right)$ is the positive constant diagonal matrix. The sliding surface rate was obtained by taking the time derivative of the sliding surface in (21) as follows:

$$
\begin{aligned}
\dot{s}=\alpha \dot{\tilde{z}}_{n}+\ddot{\tilde{z}}_{n} & =\alpha \dot{\tilde{z}}_{n}+\ddot{z}_{n}-\ddot{z}_{r_{n}} \\
& =\sigma(z)+v-\ddot{z}_{r_{n}} \\
& +\alpha \dot{\tilde{z}}_{n},
\end{aligned}
$$

where $z_{r n}=\hat{R}_{a}^{T} z_{r w n}$.

Defining the Lyapunov function candidate as

$$
V_{s}=\frac{1}{2} s^{2},
$$

the following function provides $s \rightarrow 0$ :

$$
\begin{aligned}
v=\ddot{z}_{r n}-\alpha \dot{\tilde{z}}_{n}-\mu \operatorname{sign}(s), \mu \\
=\operatorname{diag}\left(\mu_{1}, \mu_{2}\right),
\end{aligned}
$$

where the positive adaptive gain $\mu_{j}$ is greater than $\sigma_{\text {jmax }}$ in (19). Here $\mu$ was chosen as follows:

$$
\dot{\mu}_{j}=\rho_{j}\left|s_{j}\right|,
$$

where $\rho$ is a positive constant.
The control law contains the sign function; therefore, the SMC control method endures high frequency oscillations. Several methods in the literature have been proposed to solve this problem.

Property: Let us consider control $u=\bar{u}+v$ consisting of the sliding mode contouring controller based on the uncertainty dynamics and the nonlinear sliding mode controller illustrated in Figure 1. If the function $\sigma$ is upper-bounded by $\sigma_{\max }$ as in (19), and the final value of the controller gain $\mu_{j}^{*}$ in (24) satisfies $\mu_{j}^{*}>\sigma_{\text {jmax }}$, then $z_{n}$ asymptotically converges to zero, and the sliding motion is achieved. (Proof is straightforward and omitted.)

\section{Energy consumption}

The method proposed in Uchiyama et al. (2015b) was used herein to calculate the energy consumption of the feed drive system. The output power $P_{i}$ of a three-phase AC motor is given as:

$$
P_{i}=\sqrt{3} P_{f_{i}} V_{i}(t) I_{i}(t),
$$

Whereby, $V_{i}$ and $I_{i}$ are the instantaneous effective current and the voltage of a motor, respectively, and $P_{f_{i}}$ is the power factor for the $i^{\text {th }}$ axis that can be assumed constant when the load range of the motor is greater than a certain value. From (26), the energy consumption is given as:

$$
\begin{gathered}
E_{i}=\sqrt{3} P_{f_{i}} \int_{0}^{T} V_{i}(t) \cdot I_{i}(t) d t, \\
I_{i}(t)=\frac{1}{K_{\mu_{i}}}\left[l_{i} \operatorname{sign}\left(\dot{x}_{i}\right)+c_{i} \dot{x}_{i}(t)\right. \\
\left.\quad+m_{i} \ddot{x}_{i}(t)\right], \\
V_{i}(t)=I_{i}(t) Z_{i}+K_{E_{i}} \dot{x}_{i}(t),
\end{gathered}
$$

Where $K_{\mu_{i}}$ is a force is constant, $Z_{i}$ is the motor impedance and $K_{E_{i}}$ is the back-EMF coefficient. Equations (27)-(29) lead to Equation (30). Equation (30) determines the energy only from the motion trajectory and the constant.

$$
E_{i}=\sqrt{3} P_{f_{i}} \int C_{1_{i}} \ddot{x}_{i}^{2}+C_{2_{i}} \dot{x}_{i}^{2}+C_{3_{i}} \dot{x}_{i} \operatorname{sign}\left(\dot{x}_{i}\right)+C_{4_{i}}+C_{5_{i}} \ddot{x}_{i} \operatorname{sign}\left(\dot{x}_{i}\right)+C_{6_{i}} \ddot{x}_{i} \dot{x}_{i} d t,
$$




$$
\begin{aligned}
C_{1_{i}}=J_{e_{i}}^{2} \frac{Z_{i}}{K_{\mu_{i}}^{2}}, C_{2_{i}} & =B_{e_{i}}\left(\frac{Z_{i} B_{e_{i}}}{K_{\mu_{i}}^{2}}+\frac{K_{E_{i}}}{K_{\mu_{i}}}\right), C_{3_{i}}=L_{i}\left(\frac{2 Z_{i} B_{e_{i}}}{K_{\mu_{i}}^{2}}+\frac{K_{E_{i}}}{K_{\mu_{i}}}\right), C_{4_{i}}=L_{i}^{2} \frac{Z_{i}}{K_{\mu_{i}}^{2}}, C_{5_{i}} \\
& =2 L_{i} J_{e_{i}} \frac{Z_{i}}{K_{\mu_{i}}^{2}}, \quad C_{6_{i}}=J_{e_{i}}\left(\frac{2 Z_{i} B_{e_{i}}}{K_{\mu_{i}}^{2}}+\frac{K_{E_{i}}}{K_{\mu_{i}}}\right) .
\end{aligned}
$$

\section{Simulation}

A simulation was conducted based on a reference trajectory in Equation (31) and Figure 3 for the $x$ and $y$ axes to validate the effectiveness of the proposed method, $x_{r}=r \cos \left(\frac{2 \pi t}{T}\right), y_{r}=r \sin \left(\frac{2 \pi t}{T}\right)$,

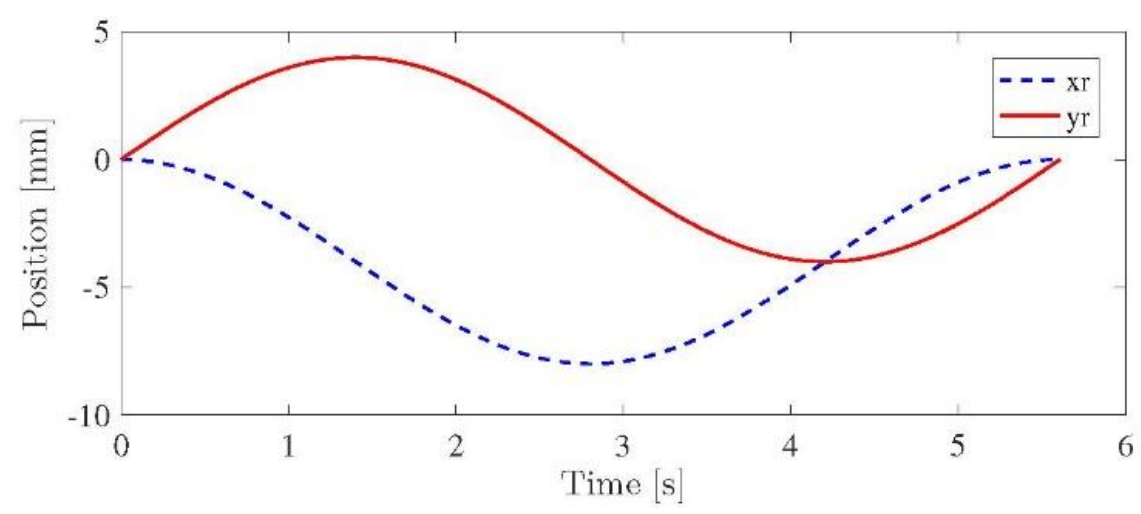

Figure 3: Reference trajectories.

Table 1: System parameters and controller parameters

\begin{tabular}{cllllllll}
\hline Axis & $m\left(\mathrm{Ns} s^{2} / \mathrm{m}\right)$ & $c(\mathrm{Ns} / \mathrm{m})$ & $l(\mathrm{~N})$ & $\lambda\left(s^{-1}\right)$ & $\begin{array}{l}\alpha \\
\left(s^{-1}\right)\end{array}$ & $\gamma\left(s^{-1}\right)$ & $\rho\left(s^{-1}\right)$ & $\beta$ \\
\hline$x$ & 88.08 & 467.20 & 45.50 & 200 & 60 & 1.2 & 0.5 & 6 \\
$y$ & 97.90 & 631.00 & 54.80 & 200 & 60 & 1.2 & 0.5 & 6 \\
\hline
\end{tabular}

A comparison with the results in Msukwa et al. (2017) was made to evaluate the performance. The proposed method includes contouring ASMC with reference adjustment and SMC based on uncertainty dynamics. The following scenarios were considered: ASMC only for tracking in each drive axis (Track), ASMC for contouring control with reference adjustment (CC), and ASMCC and uncertainty dynamics (CCU). The nonlinear second-order plant in (1) was considered as the real plant, whereas the linear model in (2) was considered as the reference plant model. The Coulomb friction force $L$ in the real plant (1) was set to vary with time as in (32) to evaluate the performance in presence of a matched uncertainty. An array of random where $r$ is the radius, and $T$ is the total time taken to complete the trajectory.

Table 1 presents the plant and controller parameters. 


\section{Simulation results}

We first evaluated the performance of the proposed approach under a low speed of 4.5 $\mathrm{mm} / \mathrm{s}$ of feed drives. Figure 4 depicts the tracking performance results in the $x$ and $y$ axes. With the Track, the average tracking error was larger in both axes compared to that of $\mathrm{CC}$ and CCU. Figure 5 shows the tracking error results in the $\sum_{n}$ coordinate frame. Both $e_{n 1}$ and $e_{n 2}$ were larger with the Track compared to that of $\mathrm{CC}$ and CCU. Accordingly, $e_{n 2}$, which was used as an estimate of the contour error $e_{c}$, was minimum when $\mathrm{CCU}$ was applied. Figure 6 illustrates the control input signals. The control input signals of the CCU were slightly larger than those of the Track and the CC only because the $\mathrm{CCU}$ generated an additional control signal $v$ to compensate for the uncertainty dynamics. The linear term $\lambda_{j}$ of the sliding surface $\bar{s}_{j}$ can be increased to allow the $\mathrm{CC}$ to achieve a contouring performance similar to that of the CCU. However, note that this will result in a higher chattering of the input signal, which will lead to a higher energy consumption.

Figure 7 exhibits the adaption of the controller gain $\mu$. The gain was initialized as $\mu_{j}(0)=0$. Moreover, the gain reached a large final value because of the adaption rule, which consequently stabilized the system. Figure 8 shows the result of the varied Coulomb friction force $L$. If we relate the findings to the results of the normal and tangential errors in Figure 5, we can see that the $\mathrm{CC}$ has a large error when the $L$ magnitude increases and almost the same error as the CCU when the $L$ magnitude is zero at $1.6 \mathrm{~s} \leq t \leq 3 \mathrm{~s}$. However, CCU had almost the same magnitude of errors all throughout, independent of the changing magnitude of $L$, because of the addition of the uncertainty compensator that compensated for these changes. Using $\mathrm{CCU}$ reduced the average contour error by $85.71 \%$. The absolute maximum contour error could be reduced by $78.64 \%$ compared with CC. Table 2 summarizes the simulation results under low speed for clarity.
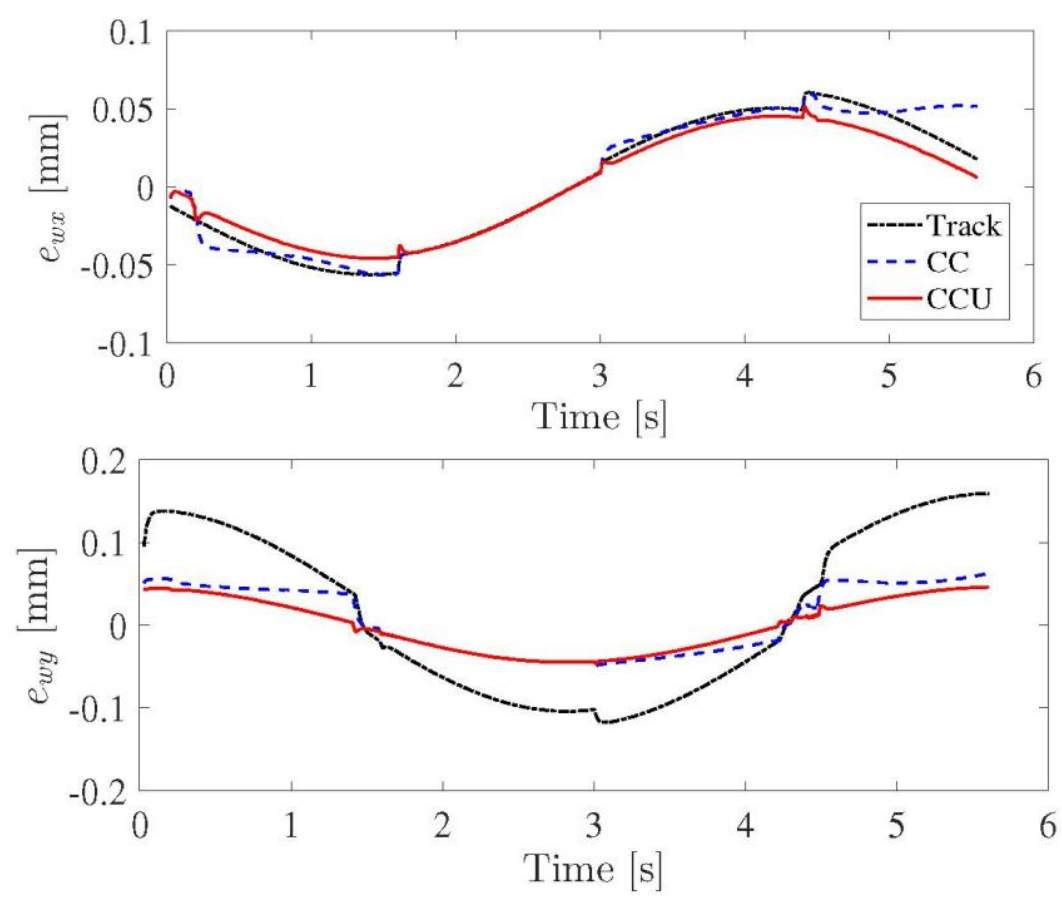

Figure 4: Tracking errors. 

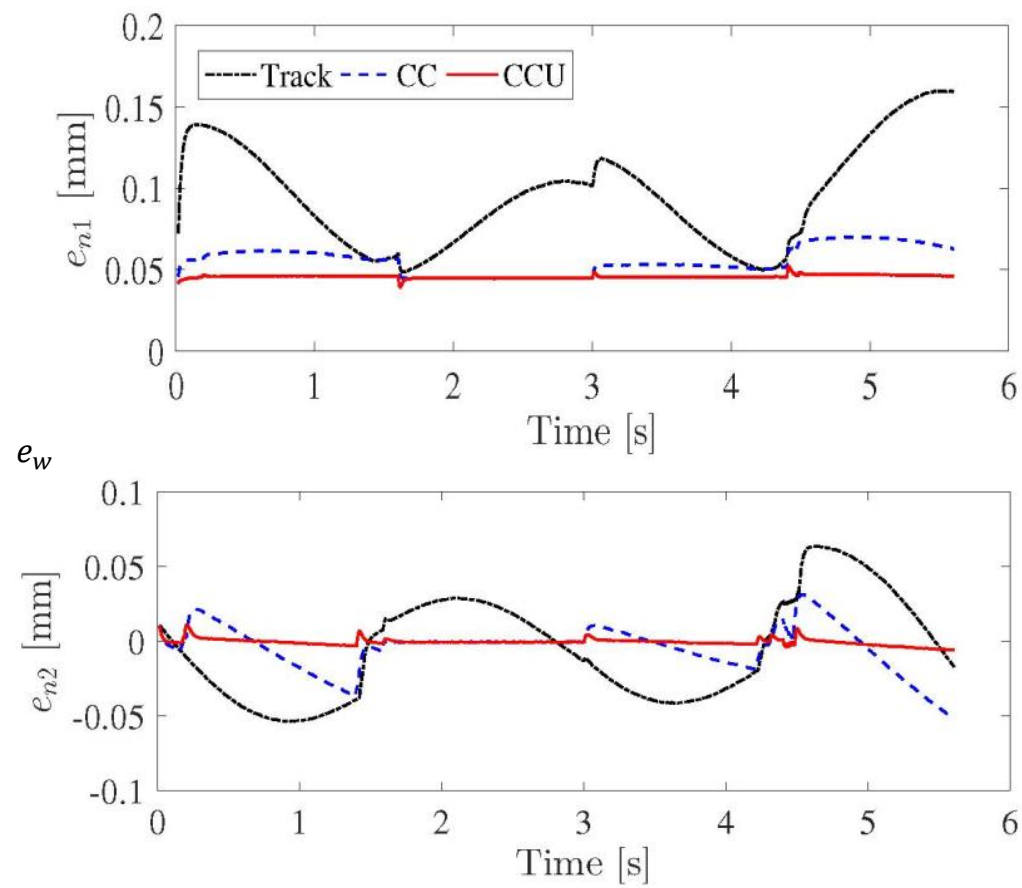

Figure 5: Tangential error and normal error $e_{n}$.
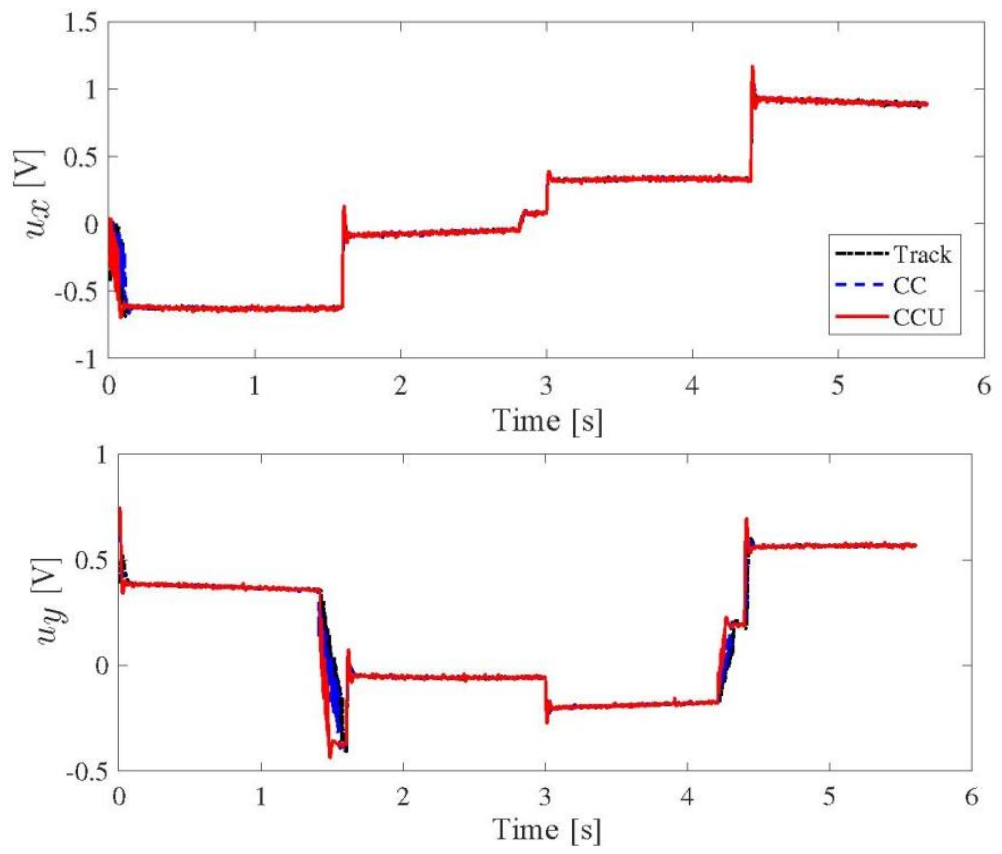

Figure 6: Control input $u$. 

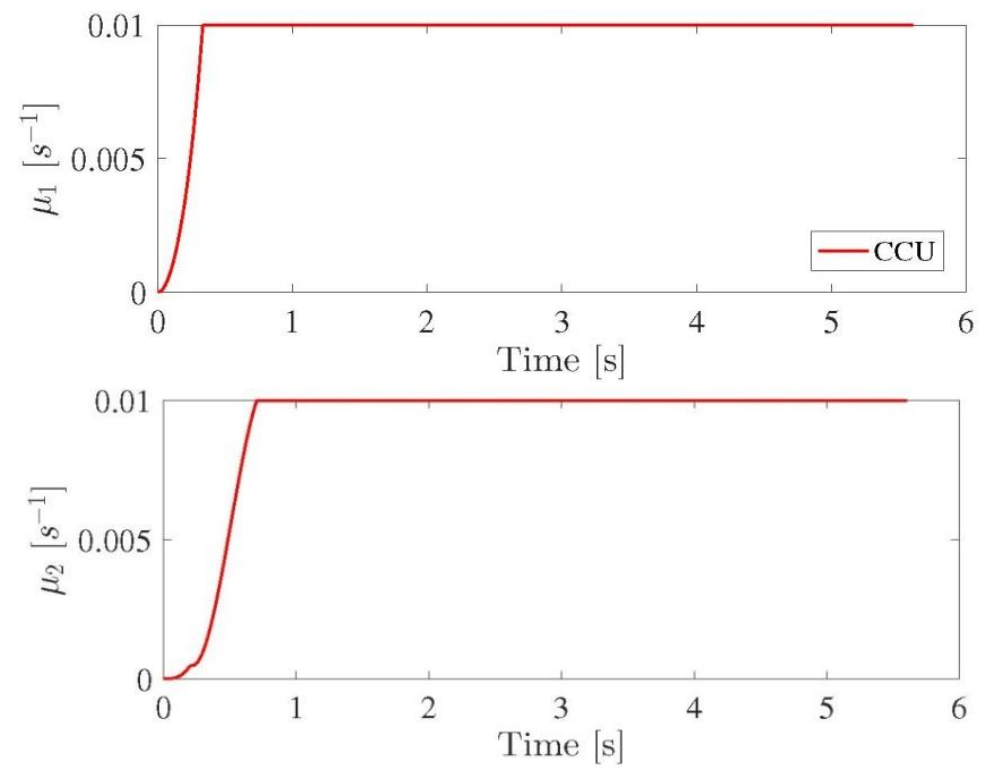

Figure 7: Controller gain $\mu$.
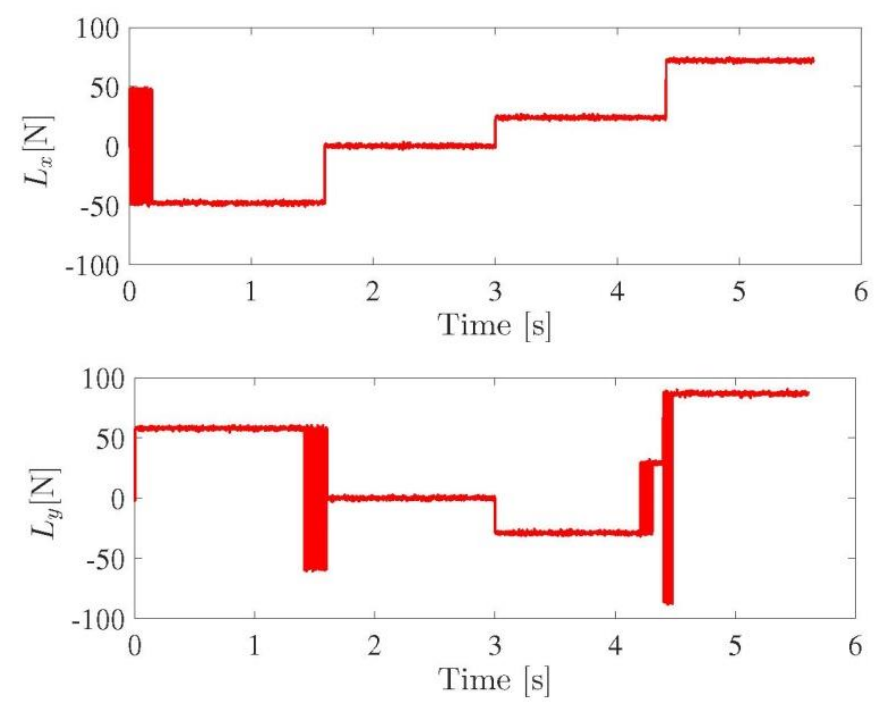

Figure 8: Coulomb friction force $L$.

Table 2: Summary of the results under low speed of $4.5 \mathrm{~mm} / \mathrm{s}$

\begin{tabular}{|c|c|c|c|c|c|}
\hline \multirow{3}{*}{ Controller } & \multicolumn{4}{|c|}{ Tangential and normal error (mm) } & \multirow{3}{*}{$\begin{array}{l}\text { Total energy } \\
\text { consumption }(\mu J)\end{array}$} \\
\hline & \multirow{2}{*}{$\frac{\text { Maximum }}{e_{n 1}}$} & \multicolumn{3}{|c|}{ Average } & \\
\hline & & $e_{n 2}$ & $e_{n 1}$ & $e_{n 2}$ & \\
\hline Track & 0.1602 & 0.0634 & 0.0947 & 0.0306 & 1.3362 \\
\hline $\mathrm{CC}$ & 0.0701 & 0.0515 & 0.0556 & 0.0112 & 1.3281 \\
\hline $\mathrm{CCU}$ & 0.0521 & 0.0110 & 0.0457 & 0.0016 & 1.3568 \\
\hline
\end{tabular}




\section{Simulation results under high speed}

We then evaluated the performance of the proposed approach under a high speed of 100 $\mathrm{mm} / \mathrm{s}$ of the feed drive. The tracking performances of both $\mathrm{CC}$ and $\mathrm{CCU}$ were almost the same. Figure 9 shows the performance in the $\sum_{n}$ coordinate frame. As with the low speed, CCU achieved the smallest error in both the $e_{n 1}$ and $e_{n 2}$ directions compared to $\mathrm{CC}$. The $e_{n}$ results when using the tracking controller were not included in Figure 9 because they were too big when high speed was used. Using CCU could reduce the average contour error by $4.48 \%$. Meanwhile, the absolute maximum contour error could be reduced by $10.13 \%$ compared to CC. In conclusion, the proposed controller can be used to increase the contouring performance in high-speed feed drive systems.
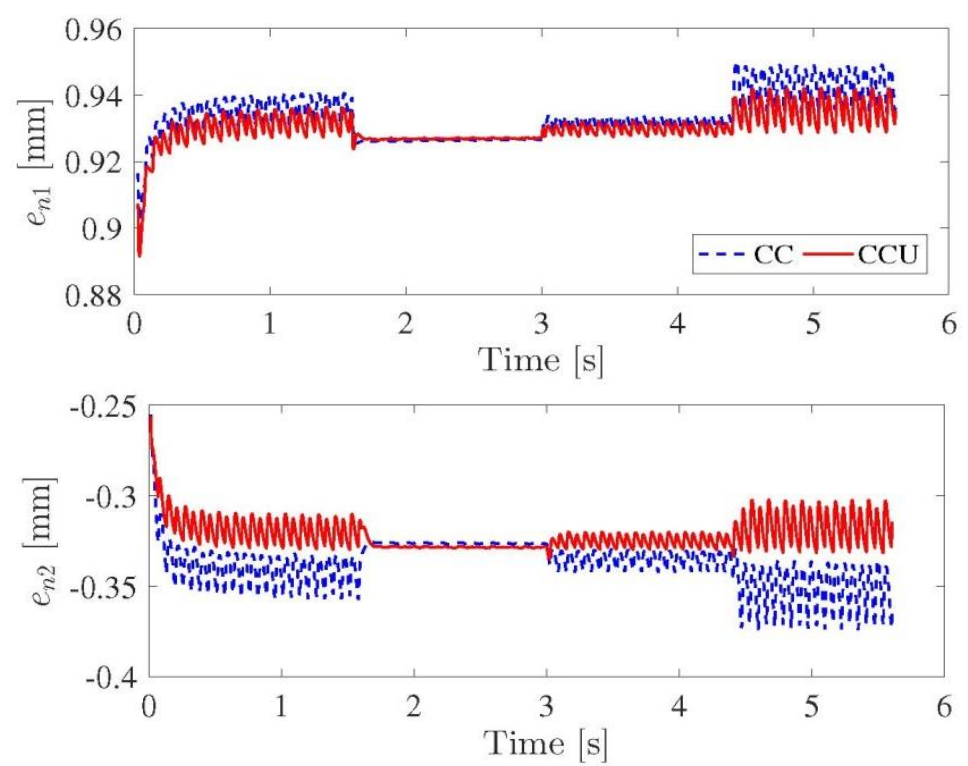

Figure 9: Tangential error and normal error $e_{n}$ under high speed.

\section{Conclusion}

This study proposed an approach to combine an adaptive sliding mode contouring controller with reference adjustment and sliding mode control based on uncertainty dynamics for the precision motion in a feed drive system. The feasibility of the approach was then demonstrated by simulation results. The proposed method showed a substantial improvement in performance by reducing the average contour error by $85.71 \%$ and the maximum contouring error by $78.64 \%$ under a low speed compared to the adaptive sliding mode contouring controller with reference adjustment. Under high speed, the proposed approach reduced the average and absolute maximum contour errors by $4.48 \%$ and $10.13 \%$, respectively. The experimental verification is left for future work.

\section{References}

Bandyopadhyay B, Deepak F and Kim KS 2009 Sliding mode control using novel sliding surfaces, 342, Lecture Notes in Control and Information Science (LNCIS), Springer, Heidelberg.

Chen Q, Tao L, Nan Y and Ren X 2016 Adaptive nonlinear sliding mode control of mechanical servo system with LuGre friction compensation. J. Dyn. Syst. Meas. Control 138(2): 1-9.

Chen SL, Liu H and Ting S 2002 Contouring control of biaxial systems based on polar coordinates. Mechatron. IEEE/ASME Trans. Mechatron. 7: 329-345.

Dong L and Tang WC 2014 Adaptive back stepping sliding mode control of flexible ball screw drives with time-varying 
parametric uncertainties and disturbances. ISA Trans. 53(1): 110-116.

Elfizy A, Bone G and Elbestawi M 2004 Model-based controller design for machine tool direct feed drives. Int. J. Mach. Tools Manuf. 44(5): 465-477.

Farrage A and Uchiyama N 2019 Design and experimental verification of adaptive sliding mode control for motion accuracy and energy saving in industrial feed drive systems. Am. Control. Conf. 1724-1729.

Farrage A and Uchiyama N 2020 Improvement of motion accuracy and energy consumption for industrial feed drive systems using adaptive sliding mode control. ISA Trans. 106: 382-391.

Ho HC, Yen JY and Lu SS 1999 A decoupled path-following control algorithm based upon the decomposed trajectory error. Int. J. Mach. Tools Manuf. 39(10): 1619-1630.

$\mathrm{Hu}$ C, Yao B and Wang Q 2010 Coordinated adaptive robust contouring controller design for an industrial biaxial precision gantry. Mechatron. IEEE/ASME Transactions 15(11): 728-735.

Ji Q, Li C, Zhu D, Jin Y, Lv Y and He J 2020 Structural design optimization of moving component in CNC machine tool for energy saving. J. Clean. Prod. 246: 118976.

Kamalzadeh A, Gordon DJ and Erkorkmaz K 2010 Robust compensation of elastic deformations in ball screw drives. Int. J. Mach. Tools Manuf. 50(6): 559-574.

Kayacan E and Peschel J 2016 Robust model predictive control of systems by modeling mismatched uncertainty. IFAC-Papers Line 49(18): 265-269.

Koren Y and Yoram 1980 Cross-Coupled Biaxial Computer Control for Manufacturing Systems. J. Dyn. Syst. Meas. Control 102(4): 265-272.

Li X, Zhao H, Zhao X and Ding H 2016 Dual sliding mode contouring control with high accuracy contour error estimation for fiveaxis CNC machine tools. Int. J. Mach. Tools Manuf. 108: 74-82.

Mohammad AEK, Uchiyama N and Sano S 2014 Reduction of electrical energy consumed by feed-drive systems using sliding-mode control with a nonlinear sliding surface. IEEE Trans. Ind. Electron. 61(6) 2875-2882.

Mohammad AEK, Uchiyama $\mathrm{N}$ and Sano $\mathrm{S}$ 2015 Energy saving in feed drive systems using sliding-mode-based contouring control with a nonlinear sliding surface. IEEE/ASME Trans. Mechatron. 20(2): 572-579.

Msukwa MR, Uchiyama N and Bui BD 2017 Adaptive nonlinear sliding mode control with a nonlinear sliding surface for feed drive systems. IEEE Int. Conf. Ind. Technol. 732-737.

Msukwa MR, Nshama EW and Uchiyama N 2019 Adaptive sliding mode control for precision motion of industrial feed drive systems with uncertainty dynamics. Am. Control Conf. 1718-1723.

Nshama, EW, Msukwa, MR and Uchiyama N 2021 A trade-off between energy saving and cycle time reduction by Pareto optimal corner smoothing in industrial feed drive systems. IEEE Access 9: 23579-23594.

Plestan F, Shtessel Y, Bregeault V and Poznyak A 2010 New methodologies for adaptive sliding mode control. Int. J. Control 83(9): 1907-1919.

Raman R, Chalanga A, Kamal S and Bandyopadhyay B 2013 Nonlinear sliding surface based adaptive sliding mode control for industrial emulator. IEEE Int. Conf. Ind. Technol. 124-129.

Ramesh R, Mannan M and Poo A 2005 Tracking and contour error control in CNC servo systems. Int. J. Mach. Tools Manuf. 45(3): 301-326.

Sencer B and Shamoto E 2013 Effective torque ripple compensation in feed drive systems based on the adaptive slidingmode controller. IEEE/ASME Trans. Mechatron. 19(6): 1764-1772.

Simba KR, Bui BD, and Msukwa MR and Uchiyama N 2018 Robust iterative learning contouring controller with disturbance observer for machine tool feed drives. ISA Trans. 75: 207-215.

Su Y, Sun D and Duan B 2005 Design of an enhanced nonlinear PID controller. Mechatronics 15(8): 1005-1024.

Uchiyama N, Ogawa Y, Abd El Khalick M 
and Sano S 2015(a) Energy saving in fiveaxis machine tools using synchronous and contouring control and verification by machining experiment. IEEE Trans. Ind. Electron. 62(9): 5608-5618.

Uchiyama N, Goto K, and Sano S 2015(b) Analysis of energy consumption in fundamental motion of industrial machines and experimental verification. Am. Control Conf. 2179-2184.

Uchiyama N, Nakamura $\mathrm{T}$ and Yanagiuchi $\mathrm{H}$ 2009 The effectiveness of contouring control and a design for three-dimensional machining. Int. J. Mach. Tools Manuf. 49(11): 876-884.

Xi XC, Zhao WS and Poo AN 2015 Improving $\mathrm{CNC}$ contouring accuracy by robust digital integral sliding mode control. Int. J. Mach. Tools Manuf. 88: 51-61.

Xu X, Gu GY, Xiong Z, Sheng X and Zhu X 2017 Development of a decentralized multi-axis synchronous control approach for real-time networks. ISA Transactions
68: 116-126.

Yang J and Altintas Y 2015 A generalized online estimation and control of five-axis contouring errors of CNC machine tools. Int. J. Mach. Tools Manuf. 88: 9-23.

Yeh S and Hsu P 2002 Estimation of the contouring error vector for the crosscoupled control design. IEEE/ASME Trans. Mechatron. 7: 44-51.

Yoon HS, Kim ES, Kim MS, Lee JY, Lee GB and Ahn SH 2015 Towards greener machine tools-A review on energy saving strategies and technologies. Renew. Sust. Energ. Rev. 48: 870-891.

Zheng J, Wang H, Man Z, Jin J and Fu M 2015 Robust motion control of a linear motor positioner using fast nonsingular terminal sliding mode. IEEE/ASME Trans. Mechatron. 20(4): 1743-1752.

Zhu B and Farouki RT 2019 A general framework for solving inverse dynamics problems in multi-axis motion control. ISA Trans. 95: 130-143. 